Nota clínica

\title{
Empleo de M-VAC en el tratamiento adyuvante del carcinoma sarcomatoide de vejiga
}

\author{
Jorge García González, Daniel Pérez Fentes*, Carlos Aliste Santos**, José M. Suárez Peñaranda**, \\ Luis León Mateos, Rafael López López
}

Servicios de Oncología Médica, *Urología y **Anatomía Patológica. Complejo Hospitalario Universitario de Santiago. Santiago de Compostela, La Coruña, España

\section{Resumen}

$\mathrm{El}$ carcinoma sarcomatoide de vejiga es una neoplasia de alto grado, que representa aproximadamente el 0,3\% de todas las neoplasias vesicales y tiene su origen en las células transicionales de la vejiga. El carcinoma sarcomatoide se caracteriza por presentar un componente epitelial y un componente sarcomatoide, consistente en células fusiformes que solamente expresan marcadores de estirpe epitelial. Presentamos el caso de una mujer de 26 años diagnosticada de un carcinoma sarcomatoide de vejiga estadio III (T3aNOMO) tratada con cistectomía parcial seguida por 4 ciclos de quimioterapia adyuvante con metotrexato, vinblastina, adriamicina y cisplatino.

Palabras clave: Carcinoma de vejiga. Carcinoma sarcomatoide. Inmunohistoquímica. Citoqueratinas. Quimioterapia.

\section{Use of M-VAC in the adjuvant treatment of sarcomatoid carcinoma of the bladder}

\section{Abstract}

Sarcomatoid bladder carcinoma is a high-grade neoplasm and accounts for approximately 0,3\% of all bladder malignancies. Sarcomatoid carcinoma originates from transitional cells of the bladder. Sarcomatoid carcinoma is charactericed by a epithelial component and a sarcomatoid component, consisting of spindle cells, that is only epithelial marker-positive. We report a 26 year-old woman diagnosed of stage III sarcomatoid bladder carcinoma (T3aNOMO) treated with partial cistectomy followed by 4 cycles of adjuvant chemotherapy with methotrexate, vinblastine, doxorubicin and cisplatin.

Keywords: Bladder carcinoma. Sarcomatoid carcinoma. Immunohistochemestry. Cytokeratins. Chemotherapy.

$\mathrm{E}$ 1 carcinoma sarcomatoide de vejiga es una variante rara del carcinoma de células transicionales de la vejiga, que se caracteriza por mostrar como componente predominante una proliferación de células fusiformes, que recuerdan un sarcoma, y cuya naturaleza epitelial se demuestra con la tinción inmunohistoquímica con citoqueratinas $^{1-5}$. El carcinoma sarcomatoide representa el $0,30 \%$ de las neoplasias de vejiga ${ }^{2}$. Su incidencia es más frecuente en varones (en una proporción de 4:1) y en las últimas décadas de la vida ${ }^{4,5}$. El curso clínico de este tumor suele ser extremadamente agresivo y el pronóstico es malo pese a la instauración de tratamiento ${ }^{1-5}$.

En este trabajo describimos el caso de una mujer joven diagnosticada de un carcinoma sarcomatoide de vejiga estadio III, que fue tratado con cistectomía parcial y quimioterapia adyuvante.

\section{CASO CLÍNICO}

Mujer de 26 años, sin antecedentes de interés, que consulta en el servicio de Urología por un cuadro de dolor suprapúbico, disuria y hematuria macroscópica total con coágulos.

En la exploración física destacaba la existencia de dolor suprapúbico a la palpación profunda, sin defensa ni otros datos de irritación peritoneal. Tanto en la palpación bimanual como a la puño percusión renal presentaba dolor en fosa lumbar derecha.

La analítica de sangre y la radiografía de tórax eran normales. El sedimento urinario estaba enmascarado por hematíes. Se realizó una ecografía abdominal con el hallazgo incidental de un hemangioma en el segmento II hepático, sin embargo el resto de la exploración fue normal. Finalmente la cistoscopia encontró una única lesión sólida, ulcerada, de unos $2,5 \mathrm{~cm}$ de diámetro máximo localizada en la cúpula vesical.

Se realizó resección transuretral en profundidad del tumor. El estudio anatomopatológico reveló un carcinoma sarcomatoide de vejiga que infiltraba la capa muscular propia (Fig. 1). El estudio inmunohistoquímico de la neoplasia demostraba positividad difusa en la gran mayoría de las células neoplásicas para 
vimentina, citoqueratinas AE1-AE3, CAM 5.2, citoqueratina 7 y positividad focal (menos del $20 \%$ de las células neoplásicas) para actina del músculo liso (Fig. 2).

El estudio de extensión de la enfermedad se completó mediante tomografía computerizada (TC) de abdomen y pelvis, el cual demostraba la presencia de un mínimo engrosamiento de la pared anterior de la cúpula vesical (Fig. 3), y gammagrafía ósea, que no objetivó lesiones metastásicas.

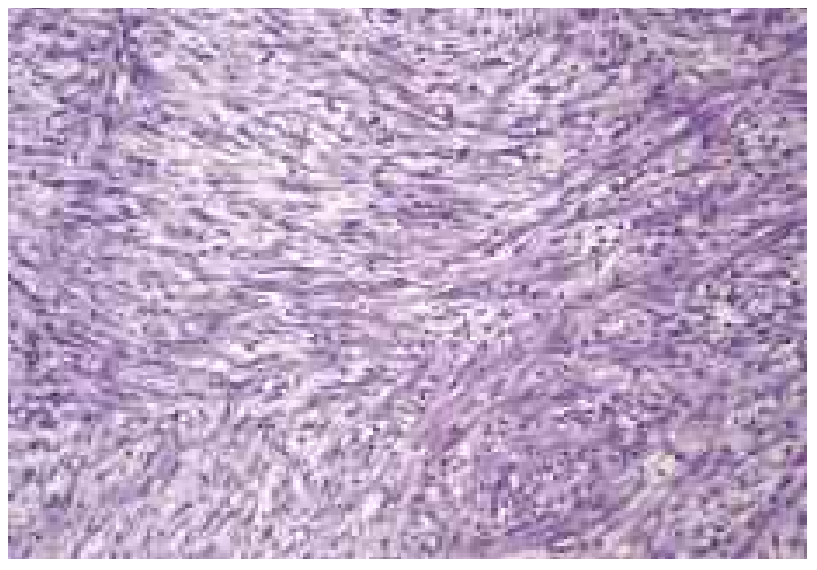

FIGURA 1
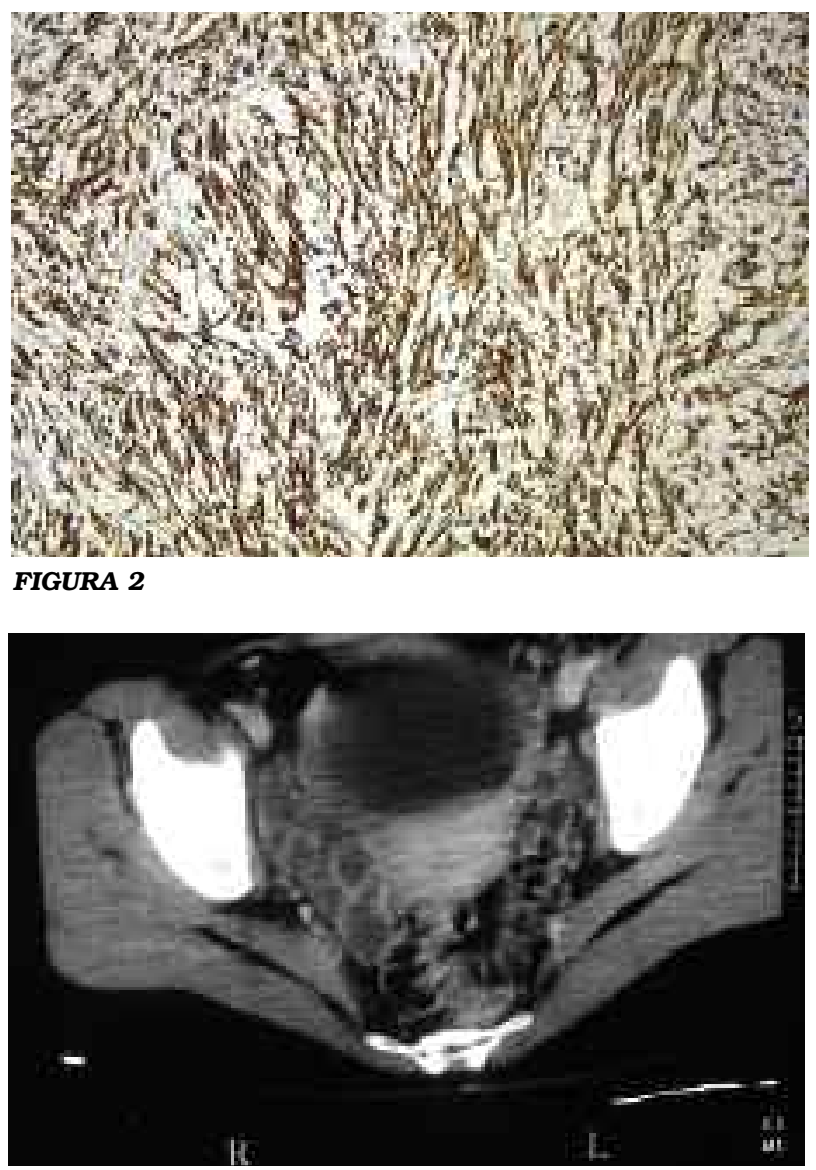

FIGURA 3
Tras descartar extensión extravesical se realizó cistectomía parcial, con resección del tumor en la cúpula vesical y del peritoneo adherido a la vejiga, así como linfadenectomía ilio-obturatriz bilateral.

La revisión anatomopatológica de la pieza quirúrgica confirmaba la existencia de un carcinoma sarcomatoide que infiltraba la capa muscular y microscópicamente la grasa perivesical. Ninguna de las 18 adenopatías aisladas a partir de las piezas de vaciamiento pélvico se encontraba infiltrada por tumor.

Con el diagnóstico de carcinoma sarcomatoide de vejiga estadio III (pT3aNOMO) la paciente se remitió al servicio de Oncología Médica para valorar tratamiento complementario.

Ofrecimos a la paciente la posibilidad de realizar seguimiento o tratamiento adyuvante con quimioterapia, decidiendo esta última opción. Así 6 semanas después de la cirugía, inició tratamiento con metotrexato, vincristina, adriamicina y cisplatino según el esquema MVAC (Tabla 1). La paciente recibió cuatro ciclos con excelente tolerancia, sin retrasos ni disminución en la dosis prevista.

Actualmente, trece meses después de finalizar el tratamiento la paciente se encuentra asintomática y sin evidencia de recidiva de la enfermedad.

\section{DISCUSIón}

Las neoplasias neoplasias vesicales en las que predomina un componente de células fusiformes son raras. Desde el punto de vista histológico se emplean dos términos que frecuentemente se confunden: carcinoma sarcomatoide y carcinosarcoma. El carcinoma sarcomatoide es una neoplasia epitelial con amplias áreas morfológicamente similares a los sarcomas, habitualmente más del 90\% de las células tumorales, pero cuya naturaleza epitelial puede demostrarse bien mediante microscopía electrónica o bien mediante inmunohistoquímica ${ }^{6}$. Por otro lado el término carcinosarcoma se emplea para tumores que presentan simultáneamente diferenciación epitelial y

Tabla 1. Muestra los fármacos que constituyen el esquema MVAC, la dosis, y los días de administración. El ciclo se repite cada 28 días

\begin{tabular}{lcc}
\hline Fármaco & Dosis & Día de administración \\
\hline Metotrexato & $30 \mathrm{mg} / \mathrm{m}^{2}$ & $1,15 \mathrm{y} 22$ \\
Vinblastina & $3 \mathrm{mg} / \mathrm{m}^{2}$ & $2,15 \mathrm{y} 22$ \\
Adriamicina & $30 \mathrm{mg} / \mathrm{m}^{2}$ & 2 \\
Cisplatino & $70 \mathrm{mg} / \mathrm{m}^{2}$ & 2 \\
\hline
\end{tabular}


verdaderamente mesenquimal entremezclándose en la mayor parte de la neoplasia ${ }^{2,6}$. Definido de este modo, el carcinoma sarcomatoide de vejiga es una neoplasia rara, que debe diferenciarse de verdaderos sarcomas y otras proliferaciones pseudosarcomatosas, como el tumor postoperatorio fusocelular y el tumor fibromixoide pseudosarcomatoso. Nuestro caso representa, indudablemente, un carcinoma sarcomatoide ya que, a pesar de no mostrar un componente epitelial, el estudio inmunohistoquímico demostró positividad para citoqueratinas. La atipia citológica y la actividad proliferativa permiten descartar las lesiones pseudosarcomatosas mencionadas con anterioridad.

El origen del carcinoma del carcinoma sarcomatoide de vejiga es todavía objeto de debate, pero estudios recientes señalan un origen monoclonal para los dos componentes habituales del tumor: el epitelial y el fusocelular; y que una divergencia clonal da lugar a ambos en momentos más tardíos de su progresión o diferenciación ${ }^{7}$.

Los síntomas más comunes del carcinoma sarcomatoide son hematuria macroscópica (57\%) o microscópica $(28 \%)$, disuria $(28 \%)$ y polaquiuria $(21 \%)^{4}$.

La conducta terapéutica en estas neoplasias no está estandarizada debido a su baja incidencia. De todos modos, en la mayoría de los casos publicados la cistectomía radical es el tratamiento de elección, seguida generalmente de radioterapia o quimioterapia $^{5,8}$. Sin embargo, el beneficio del tratamiento complementario frente a la cirugía sola en cuanto a disminución del riesgo de recidiva y supervivencia global se desconoce ${ }^{8}$.

En relación al empleo de quimioterapia se ha comunicado un caso de carcinoma sarcomatoide de vejiga con metástasis pulmonares en el que tras tratamiento paliativo con cisplatino y gemcitabina se alcanzó respuesta completa de las lesiones neoplási$\operatorname{cas}^{9}$. Además, Antonelli et al. comunicaron un caso de un varón de 34 años diagnosticado de un carcinoma sarcomatoide de vejiga con metástasis ganglionares, hepáticas y pulmonares, en el que se alcanzó respuesta completa tras realizar tratamiento neoadyuvante con adriamicina e ifosfamida, cirugía radical y quimioterapia adyuvante con los mismos fármacos ${ }^{10}$.

El pronóstico del carcinoma sarcomatoide de vejiga es malo. Su supervivencia media es de 9.8 meses, siendo el estadio del tumor al diagnóstico el factor pronóstico más importante ${ }^{3-4}$.
En nuestro caso, debido a la edad de la paciente y a la histología de mal pronóstico, decidimos ofrecer tratamiento citotóxico complementario con cuatro ciclos de M-VAC, pues este esquema de quimioterapia ha demostrado disminuir el riesgo de recidiva en pacientes con carcinoma de células transicionales de vejiga localmente avanzado ${ }^{11,12}$.

En conclusión, ante la falta de un tratamiento estándar en el manejo del carcinoma sarcomatoide de vejiga debe individualizarse la indicación del tratamiento complementario con radioterapia y quimioterapia.

\section{REFERENCIAS}

1. Young RH, Wick MR, Mills SE. Sarcomatoid carcinoma of the urinary bladder. A clinicopathologic analysis of 12 cases and review of the literature. Am J Clin Pathol. 1988;90(6): 653-661.

2. Torenbeek R, Blomjous CE, Van der Valk P, Meijer CJ. Sarcomatoid carcinoma vs carcinosarcoma of the urinary bladder. Am J Surg Pathol. 1994; 18 (3):241-249.

3. Lopez-Beltran A, Pacelli A, Rothenberg HJ, Wollan PC, Zincke H, Blute ML, et al. Carcinosarcoma and sarcomatoid carcinoma of the bladder: clinicopathological study of 41 cases. J Urol. 1998; 159(5):1497-1503.

4. Ikegami H, Iwasaki H, Ohjimi Y, Tekeuchi T, Ariyoshi A, Kikuchi M. Sarcomatoid carcinoma of the urinary bladder: a clinicopathological and immunohistochemical analysis of 14 patients. Hum Pathol. 2000;31(3) 332-340

5. Spiess PE, Tuziak T, Tibbs RF, Bassett R, Tamboli P, Brown GA Grossman HB, Ayala AG, Czerniak B. Pseudosarcomatous and sarcomatous proliferations of the bladder. Hum Pathol. 2007;38 (5):753-761.

6. Reuter VE. Sarcomatoid lesions of the urogenital tract. Semin Diagn Pathol. 1993 May;10(2):188-201.

7. Sung MT, Wang M, MacLennan GT, Eble JN, Tan PH, Lopez-Beltran A, Montironi R, Harris JJ, Kuhar M, Cheng L. Histogenesis of sarcomatoid urothelial carcinoma of the urinary bladder: evidence for a common clonal origin with divergent differentiation. J Pathol. 2007;211(4):420-30.

8. Ogishima T, Kawachi Y, Saito A, Suzuki F, Ishi F, Tanaka T. Sarcomatoid carcinoma and carcinosarcoma of the urinary bladder. Int $J$ Urol 2002; 9(6):354-358.

9. Froehner M, Gaertner HJ, Manseck A, Wirth MP. Durable complete remission of metastatic sarcomatoid carcinoma of the bladder with cisplatin and gemcitabine in an 80-year-old man. Urology. 200;58(5):799.

10. Antonelli A, Simeone C, Ferrari V, Tardanico R, Cunico SC. Durable and complete remission of a metastatic bladder sarcomatoid carcinoma with chemotherapic and surgical treatments. Arch Ital Urol Androl. 2006;78 (2):67-70.

11. Stöckle M, Meyemburg W, Wellek S, Voges GE, Gertembach U, Thüroff JW, et al. Advanced bladder cancer (stages T3b, T4a, pN1 and pN2): improved survival after radical cystectomy and 3 adjuvant cycles of chemotherapy. Results of a controlled prospective study. J Urol. 1992;148 (2 Pt 1):302-6; discussion 306-307.

12. Lehman J, Franzaring L, Thüroff J, Wellek S, Stökle M. Complete longterm survival data from data from a trial of adjuvant chemotherapy vs control after radical cystectomy for locally advanced bladder cancer. BJU Int. 2006;97(6):42-47.

Correspondencia autor: Dr. Jorge García González

Servicio de Oncología Médica.

Complejo Hospitalario Universitario de Santiago.

Travesa da Choupana $\mathrm{s} / \mathrm{n}$

Santiago de Compostela 15706 La Coruña. Tel.: 981950544

E-mail autor: jorgejgglez@yahoo.es

Información artículo: Nota clíncia

Trabajo recibido: septiembre 2007

Trabajo aceptado: octubre 2007 